\title{
Intrinsic antiviral immunity drives neurodegeneration in Alzheimer disease
}

\author{
Stefano Pluchino and Cory Willis \\ Department of Clinical Neurosciences and NIHR Biomedical Research Centre, University of Cambridge, Cambridge, United Kingdom.
}

\begin{abstract}
$\beta$-Amyloid aggregates found in brain plaques are viewed as triggers of cytotoxicity and neuroinflammation in Alzheimer disease (AD). However, the main $\beta$-amyloid $(A \beta)$ species and what imbues the aggregates with such toxic potential are still not yet understood. In this issue of the $J C l$, Roy et al. show that $A \beta$ complexed with nucleic acids triggers an antiviral type I interferon response in neuroglia, resulting in complement-mediated synapse elimination in AD models. These findings identify a putative endogenous immune signaling axis that drives neurodegeneration in $A D$ and has strong implications for the development of precise therapeutic strategies.
\end{abstract}

\section{A role for type I IFNs}

The identification of a role for immune cells in the progression of Alzheimer disease (AD) has led to intense focus on the adaptive (1) and innate branches of the immune system (2). Microglia are the main innate immune cells present in the brain and are equipped with the requisite classes of signalling receptors to sense and perpetuate local inflammation (3).

When intracellular innate immune sensors recognize viral or self-derived nucleic acids (NAs), an activated antiviral state produces type I interferon (IFN) and induces interferon-stimulated genes (ISGs). Type I IFN signalling also mediates neuroinflammation in $\mathrm{AD}$, both in animal disease models and human subjects (4), which implies that this signalling pathway activates as an integral response to disease progression.

In the $\mathrm{AD}$ brain, the accumulation of aggregated $\beta$-amyloid $(A \beta)$ fibrils into $\mathrm{AD}$ plaques occurs in concomitance with a chronic neuroinflammatory response (5). This plaque-inflammatory response includes the induction of ISGs, reactive microgliosis, and astrogliosis, as well as increased proinflammatory cytokine production (5). Soluble $A \beta$ oligomers bind with negatively charged factors, such as NAs, thus expediting the formation of insoluble amyloid fibrils (6). Importantly, NA-containing amyloid fibrils potently activate dendritic cells and enable type I IFN production to stimulate systemic autoimmunity in mice (7)

In this issue of the JCI, Roy et al. set out to determine if NA-containing $\left(\mathrm{NA}^{+}\right)$ amyloid fibrils were able to elicit sufficient production of type I IFN to drive neuroinflammation and neurodegeneration in the AD brain (8).

The authors examined the transcriptional profile of a brain region crucial for human memory and the main target in AD - the hippocampus. They used a series of animal models for AD (APP ${ }^{\mathrm{NL}-\mathrm{G}-\mathrm{F}}, 5 \mathrm{XFAD}$, APP;tTa, and APP-PS1) and showed increased expression of gene markers for microglia (Aifl) and astrocytes (Gfap) These changes were paralleled by upregulation of genes that were induced by type

Related Article: p. 1912

Conflict of interest: SP is cofounder and chief scientific officer at Cambridge Innovation Technologies Consulting (CITC) Ltd. and iSTEM Therapeutics and cofounder and non-executive director at Asitia Therapeutics. Copyright: (c) 2020, American Society for Clinical Investigation.

Reference information: / Clin Invest. 2020;130(4):1622-1624. https://doi.org/10.1172/JCI135906.
I IFNs (i.e., Irf7, Cxcl10, Oas1) as well as AD-related proinflammatory factors (i.e., C3, Tnf, Il1 $\beta$ ) (8), as shown in previous studies (4).

The in vitro exposure of mixed glia cultures to $\mathrm{NA}^{+}$generic amyloid induced a type I ISG signature and led to increased IFN- $\beta$ secretion in tissue-culture supernatants. This antiviral immune response was markedly reduced when mixed glia cultures were pretreated with a drug that depleted the culture of microglia (liposome-encapsulated clodronate). These results suggest that $\mathrm{NA}^{+}$amyloid triggers the activation of type I IFN signaling predominantly in microglia. In vivo, the transcriptional analysis of the hippocampi from wild-type (WT) mice injected with $\mathrm{NA}^{+}$generic amyloid revealed an activation profile comparable to that of the experimental AD brains, as above. Indeed, $\mathrm{NA}^{+}$generic amyloid triggered antiviral responses onto microglial and astrocytes and in vivo, when injected into the brain parenchyma (8).

Further in vivo validation in the 5XFAD mouse brain (9) revealed that the vast majority of $\mathrm{A} \beta$ plaques contained NA inclusions, and that their frequency increased with age. Systematic analysis of these amyloid plaques demonstrated a substantial increase of reactive, phagocytic microglia in close proximity to $\mathrm{NA}^{+}$ plaques, whereas microglia near to $\mathrm{NA}^{-}$ plaques remained nonphagocytic. $\mathrm{NA}^{+}$ plaque-associated microglia exclusively expressed the microglial neurodegenerative phenotype (MGnD) marker Clec7a (10), whereas a complete absence of Clec7a expression was observed in microglia associated with $\mathrm{NA}^{-}$plaques. These data suggest the coexistence of $\mathrm{NA}^{+}$plaques and the induction of type I IFN signalling in a subset of (plaque-associated) MGnD that constitute an integral element in the propagation of innate inflammatory responses in the $\mathrm{AD}$ brain. While confirming an upregulation of the astroglial marker Gfap, this in vivo part of the study 


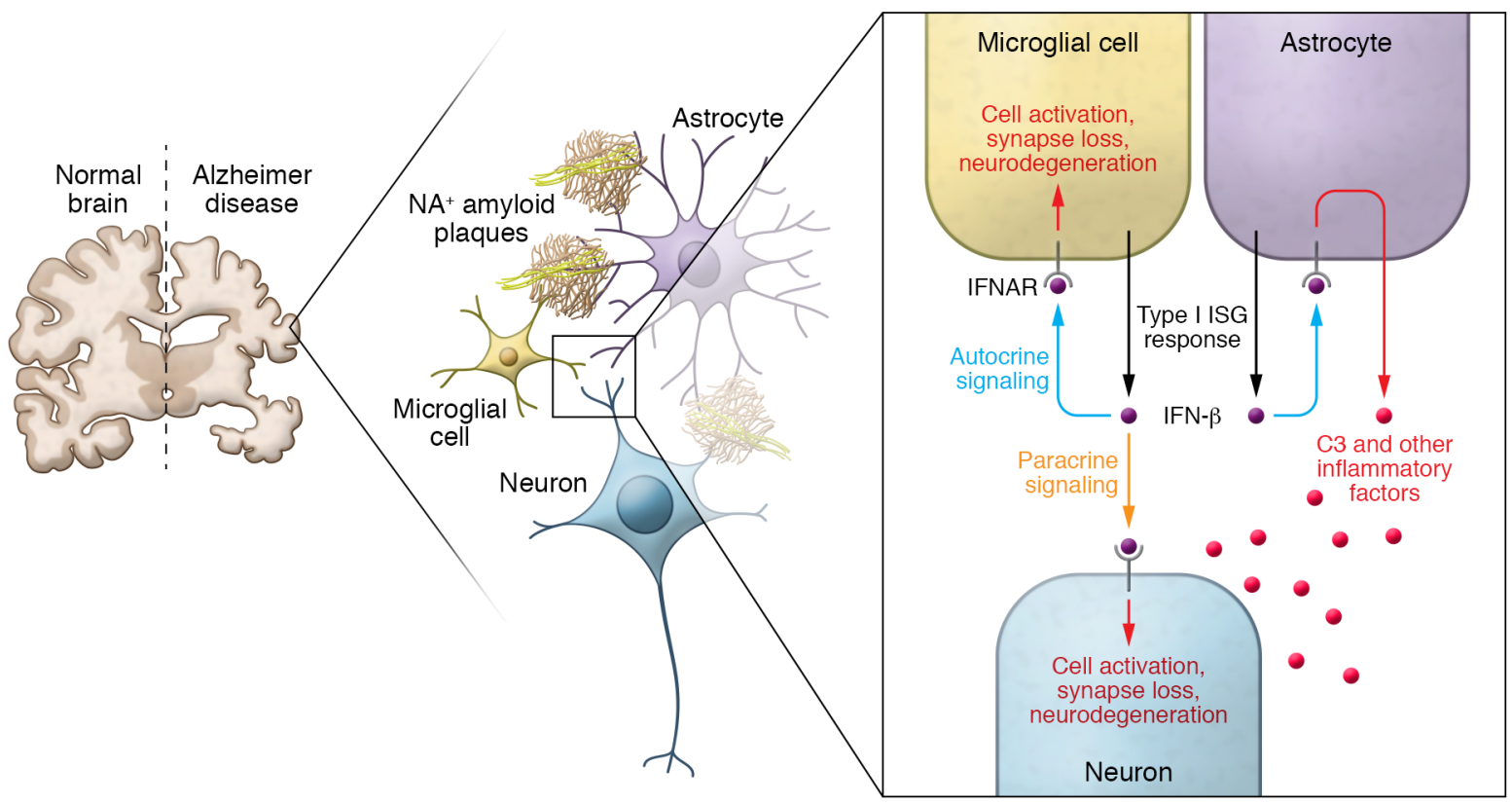

Figure 1. Model of type I IFN-mediated neuroinflammation and synapse loss in AD. NA ${ }^{+}$amyloid induces a type I ISC signature leading to increased IFN- $\beta$ secretion, predominantly in microglia. Type I IFNs act via IFN- $\alpha / \beta$ receptors (IFNARs) to induce type I IFNs and AD-related proinflammatory factors, such as C3 in astrocytes. Other microglia gene markers are also upregulated. Autocrine and paracrine signaling perpetuates cell activation and drives synapse loss and neurodegeneration.

failed to address the spatial organization of astrocytes in respect to $\mathrm{A} \beta$ plaques and NA inclusions (8).

\section{Neuroinflammation and synapse loss}

Microglia, which mediate synaptic pruning during brain development $(11,12)$, are also responsible for synapse loss in neurodegeneration (10). To identify a mechanism linking neuroinflammation with synapse loss in $\mathrm{AD}$, Roy and colleagues injected a potent type I IFN recombinant interferon- $\beta$ (rIFN- $\beta$ ) into the brains of WT mice and evaluated the cellular and molecular effectors that regulate several innate immune responses (13), including microglia activation (14). Intracerebroventricular (i.c.v.) injection of rIFN- $\beta$ induced the expression of genes associated with the MGnD phenotype and triggered a cellular response predominantly characterized by increased reactive, phagocytic microglia engulfing synaptic puncta. Conversely, injecting an $\alpha$ IFN- $\alpha / \beta$ receptor ( $\alpha$ IFNAR) blocking antibody into young (3-month-old) 5XFAD mice suppressed type I IFN signalling in reactive microglia, reduced Clec7a expression, and restored synaptic puncta density to control levels. Similar changes in microgliosis were also identified in aged (10- to 12-month old) APP NL-G-F mice injected with $\alpha$ IFNAR blocking antibody. Interestingly, $\alpha$ IFNAR blockade failed to alter plaque load in both young 5XFAD and aged APP ${ }^{\text {NL-G-F }}$ mice (8). Thus, type I IFNs act via IFN $-\alpha / \beta$ receptors on microglia and signal both autocrine and paracrine pathways to perpetuate cell activation and drive synapse loss and neurodegeneration in the $\mathrm{AD}$ brain.

\section{The $\mathrm{NA}^{+}$amyloid-type I IFN-C3 signaling axis}

Complement component 3 (C3) is recognized as a central mediator in the pruning of weak synapses tagged with complement proteins by microglial cells expressing complement receptors during development $(11,12)$. Complement proteins are also substantially upregulated during aging and neurodegeneration - even prior to any evidence of neuronal loss, which anticipates a role for complement-mediated mechanisms in neuronal dysfunction and loss (15). Furthermore, aging promotes sustained expression of type I IFN in the brain, which induces an inflammatory microglia phenotype and leads to increased production of complement factors (16).

What remains to be established is whether signaling through IFN- $\alpha / \beta$ recep- tor induces the expression of complement proteins. To this aim, the authors injected stereotaxically rIFN- $\beta$ into the hippocampi of WT mice and measured C3 at transcript and protein levels. Interestingly, rIFN- $\beta$ increased $\mathrm{C} 3$ expression in astrocytes only. Conversely, the injection of $\mathrm{NA}^{+}$generic amyloid into the hippocampi of WT mice elicited a substantial induction of the expression of complement genes. The i.c.v. injection of an aIFNAR attenuated the complement response, strongly suggesting that type I IFNs signal through IFN- $\alpha / \beta$ receptor onto astrocytes for complement activation in response to $\mathrm{NA}^{+}$amyloid in AD. The authors confirmed these findings in the 5XFAD and APP ${ }^{\mathrm{NL}-\mathrm{G}-\mathrm{G}}$ mouse AD models, where blocking $\alpha$ IFNAR with an antibody reduced $\mathrm{C} 3$ protein and transcript expression, respectively. While $\mathrm{NA}^{+}$ amyloid as well as the recombinant protein stimulated type I IFN signaling in both in microglia and astrocytes, downstream complement activation occurred only in astrocytes (8). Therefore, the model proposed here suggests that $\mathrm{NA}^{+}$amyloid is able to induce $\mathrm{C} 3$ production in astrocytes but not in microglia (Figure 1).

In experimental demyelination in mice, C3 but not C1q has recently emerged as a major driver of microglia engulfment and 
elimination of synapses (17). To address whether C3 was indeed necessary for the type I IFN-triggered synapse elimination in $\mathrm{AD}$, the authors injected cerebral ventricles of $C 3^{-/-}$mice with rIFN- $\beta$ and quantified microglia and synapses. Interestingly, while $\mathrm{C3}^{-/-}$microglia exhibited a reactive morphology, $\mathrm{C3}^{-/-}$brains were completely protected from postsynaptic puncta loss after rIFN- $\beta$ compared with controls (8).

Together, these results highlight a critical $\mathrm{NA}^{+}$amyloid-type I IFN complement-mediated signaling axis that triggers and perpetuates neuroglial reactivity and drives synapse loss in AD.

\section{Conclusion}

The work by Roy et al. (8) proposes a role for $\mathrm{NA}^{+}$amyloid as a potent inducer of type I IFN signalling and complement-mediated synapse elimination by microglia and astrocytes in $\mathrm{AD}$ and potentially other neurodegenerative disorders. At the same time, the study raises a number of questions warranting further investigation.

Further studies are required to establish the specificity and the main source of cell-free NAs (i.e., DNA, RNA), and whether these might represent a potential disease biomarker to be detected and quantified in biological fluids (18). Additional mechanistic work is also indispensable to identify the key components of this antiviral type I IFN-mediated neuroglia reaction, and to address the relative contribution of the autocrine versus paracrine signaling pathways - both mediated by soluble factors and associated with extracellular membrane vesicles (19) - in the perpetuation of neuroinflammation in AD. Nonetheless, it will also be important to determine if this endogenous type I IFN-driven response to $\mathrm{NA}^{+}$amyloid, and its consequences on excessive synapse elimination and neurodegeneration, plays any role in non-AD chronic neurological conditions associated with increased type I IFN serology (20).
In summary, Roy et al. provide compelling evidence that neuroinflammation is a critical feature of AD pathobiology. While demonstrating that $\mathrm{NA}^{+}$-containing amyloid is a potent inducer of type I IFN signalling in neuroglia, this study also addresses the key mechanisms of the induction and propagation of antiviral innate immune responses in the brain, which in turn amplify complement-mediated synapse loss and neurodegeneration (8).

These results not only expand our understanding of the pathology in $\mathrm{AD}$ but also identify a putative endogenous immune signaling axis driving neuroinflammation and neurodegeneration in $\mathrm{AD}$, and may have strong implications for the development of precise therapeutic strategies.

\section{Acknowledgments}

The authors acknowledge current members of the Pluchino laboratory, who contributed to (or inspired) this commentary.

Address correspondence to: Stefano Pluchino, Clifford Allbutt Building, Cambridge Biosciences Campus, Hills Road, CB2 OHA Cambridge, United Kingdom. Phone: 44.1223.331163; Email: spp24@ cam.ac.uk.

1. Gate D, et al. Clonally expanded CD8 T cells patrol the cerebrospinal fluid in Alzheimer's disease. Nature. 2020;577(7790):399-404.

2. Taylor JM, Moore Z, Minter MR, Crack PJ. Type-I interferon pathway in neuroinflammation and neurodegeneration: focus on Alzheimer's disease. J Neural Transm (Vienna). 2018;125(5):797-807.

3. Olson JK, Miller SD. Microglia initiate central nervous system innate and adaptive immune responses through multiple TLRs. JImmunol. 2004;173(6):3916-3924.

4. Taylor JM, Minter MR, Newman AG, Zhang M, Adlard PA, Crack PJ. Type-1 interferon signaling mediates neuro-inflammatory events in models of Alzheimer's disease. Neurobiol Aging. 2014;35(5):1012-1023.

5. Scheltens P, et al. Alzheimer's disease. Lancet. 2016;388(10043):505-517.
6. Di Domizio J, et al. Binding with nucleic acids or glycosaminoglycans converts soluble protein oligomers to amyloid. J Biol Chem. 2012;287(1):736-747.

7. Di Domizio J, et al. Nucleic acid-containing amyloid fibrils potently induce type I interferon and stimulate systemic autoimmunity. Proc Natl Acad Sci USA. 2012;109(36):14550-14555.

8. Roy ER, et al. Type I interferon response drives neuroinflammation and synapse loss in Alzheimer disease. J Clin Invest. 2020;130(4):1912-1930.

9. Oakley $\mathrm{H}$, et al. Intraneuronal beta-amyloid aggregates, neurodegeneration, and neuron loss in transgenic mice with five familial Alzheimer's disease mutations: potential factors in amyloid plaque formation. J Neurosci. 2006;26(40):10129-10140.

10. Krasemann S, et al. The TREM2-APOE pathway drives the transcriptional phenotype of dysfunctional microglia in neurodegenerative diseases. Immunity. 2017;47(3):566-581.e9.

11. Filipello F, et al. The microglial innate immune receptor TREM2 is required for synapse elimination and normal brain connectivity. Immunity. 2018;48(5):979-991.e8.

12. Paolicelli RC, et al. Synaptic pruning by microglia is necessary for normal brain development. Science. 2011;333(6048):1456-1458.

13. Akira S, Uematsu S, Takeuchi O. Pathogen recognition and innate immunity. Cell. 2006;124(4):783-801.

14. Song WM, Colonna M. The identity and function of microglia in neurodegeneration. Nat Immunol. 2018;19(10):1048-1058.

15. Hong S, et al. Complement and microglia mediate early synapse loss in Alzheimer mouse models. Science. 2016;352(6286):712-716.

16. Deczkowska A, et al. Mef2C restrains microglial inflammatory response and is lost in brain ageing in an IFN-I-dependent manner. Nat Commun. 2017;8(1):717.

17. Werneburg S, et al. Targeted complement inhibition at synapses prevents microglial synaptic engulfment and synapse loss in demyelinating disease. Immunity. 2020;52(1):167-182.e7.

18. Pös O, Biró O, Szemes T, Nagy B. Circulating cell-free nucleic acids: characteristics and applications. Eur J Hum Genet. 2018;26(7):937-945.1.

19. Cossetti C, et al. Extracellular vesicles from neural stem cells transfer IFN- $\gamma$ via Ifngr1 to activate Stat1 signaling in target cells. Mol Cell. 2014;56(2):193-204.

20. Labzin LI, Heneka MT, Latz E. Innate immunity and neurodegeneration. Annu Rev Med. 2018;69:437-449. 